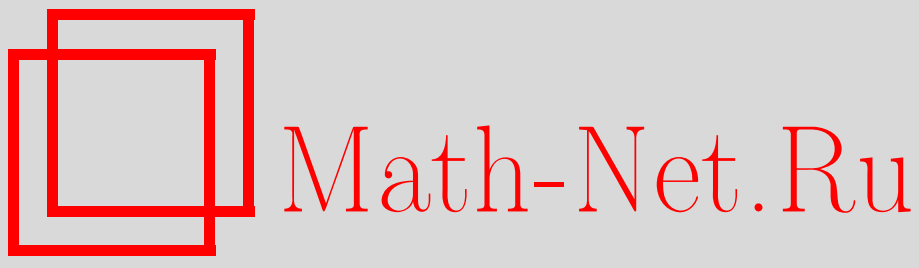

С. И. Дудов, И. В. Златорунская, Равномерная оценка выпуклого компакта шаром произвольной нормы, Матем. сб., 2000, том 191, номер 10, 13-38

DOI: https://doi.org/10.4213/sm513

Использование Общероссийского математического портала Math-Net.Ru подразумевает, что вы прочитали и согласны с пользовательским соглашением http: //www. mathnet.ru/rus/agreement

Параметры загрузки:

IP: 54.198 .55 .26

26 апреля 2023 г., $06: 53: 27$ 
УДК $517.982 .256+519.853 .3$

\author{
С.И. Дудов, И.В. Златорунская
}

\title{
Равномерная оценка выпуклого компакта шаром произвольной нормы
}

\begin{abstract}
Рассматривается задача о равномерной оценке (наилучшем приближении) выпуклого компакта шаром произволной нормы в метрике Хаусдорфа, порожденной используемой нормой.

Задача редуцирована к одной задаче выпуклого программирования, что позволило использовать при ее исследовании средства вьпуклого анализа. Получены необходимые и достаточные условия решения задачи и некоторые его свойства. В частности, доказано, что центр хотя бы одного шара наилучшего приближения содержится в оцениваемом компакте, а также получены условия, при которых все множество центров шаров наилучшего приближения содержится в этом компакте, и условие единственности решения.

Библиография: 19 названий.
\end{abstract}

\section{§1. Введение}

Получение оценок и аппроксимаций достаточно сложных множеств множествами простой структуры является одним из направлений негладкого анализа. Такие задачи находят обширные приложения в естествознании и прежде всего в самой математике. Можно указать на многочисленные работы, связанные с внешними и внутренними эллипсоидальными оценками множеств и многозначных отображений (см., например, [1]). Наряду с эллипсоидом к числу наиболее простых множеств как в геометрическом смысле, так и по числу задающих параметров относится шар любой нормы.

Рассмотрим следующую задачу. Пусть $D$ - подлежащий оценке заданный вьпуклый компакт из конечномерного действительного пространства $\mathbb{R}^{p}$, функция $n(x)$ удовлетворяет на $\mathbb{R}^{p}$ аксиомам нормы, $\rho(A, B)=\sup _{x \in A} \inf _{y \in B} n(x-y)$ - уклонение множества $A$ от множества $B$ в норме $n(\cdot), \quad h(A, B)=$ $\max \{\rho(A, B) ; \rho(B, A)\}-$ расстояние Хаусдорфа между множествами $A$ и $B$ в норме $n(\cdot), B n(x, r)=\left\{y \in \mathbb{R}^{p}: n(x-y) \leqslant r\right\}-$ шар в норме $n(\cdot)$ с центром в точке $x$ и радиусом $r$. Тогда задачу о наилучшем приближении выпуклого компакта $D$ шаром нормы $n(\cdot)$ в метрике Хаусдорфа, порожденной этой нормой, можно записать в виде

$$
h(D, B n(x, r)) \rightarrow \min _{x \in \mathbb{R}^{p}, r>0} .
$$

Задача (1.1) рассматривалась в работе М. С. Никольского и Д. Б. Силина [2] для случая, когда $n(\cdot)$ - евклидова норма. В этой работе доказаны сушествование и

Работа вьполнена при поддержке Российского фонда фундаментальных исследований (грант № 98-01-00048) и программы "Ведущие научные школы" (грант № 00-15-96123).

$$
\text { (C) С. И. ДУДОВ, И. В. ЗЛАТОРУНСКАЯ }
$$


единственность решения, получены необходимое условие решения и его свойства. Там же отмечено, что полученные результаты нетрудно перенести на случай более обшей “эллипсоидальной” нормы ввиду простой связи решений задач для этих норм.

Цель данной работы - исследование задачи (1.1) для произвольной используемой нормы $n(\cdot)$.

На задачу (1.1) интересно посмотреть в сравнении с задачей о построении шара нормы $n(\cdot)$ с наименьшим радиусом, содержашего компакт $D$ :

$$
R(x) \equiv \max _{y \in D} n(x-y) \rightarrow \min _{x \in \mathbb{R}^{p}}
$$

и задачей о построении шара наибольшего радиуса, содержашегося в $D$,

$$
\rho_{\Omega}(x) \equiv \min _{y \in \Omega} n(x-y) \rightarrow \max _{x \in D}
$$

Здесь $\Omega=\overline{\mathbb{R}^{p} \backslash D}$ - замыкание дополнения множества $D$ до $\mathbb{R}^{p}$. Задачи $(1.2)$ и (1.3) являются соответственно задачами о внешней и внутренней оценке множества $D$ шаром нормы $n(\cdot)$. С этой точки зрения задачу $(1.1)$ можно назвать задачей о равномерной оценке.

Для случая евклидовой нормы, как доказано в [2], шар наилучшего приближения является единственным, а его центр $x_{0}$ содержится в $D$ и является единственным решением задачи

$$
R(x)-\rho_{\Omega}(x) \rightarrow \min _{x \in D}
$$

При этом радиус искомого шара есть $r_{0}=\left(R\left(x_{0}\right)+\rho_{\Omega}\left(x_{0}\right)\right) / 2, \mathrm{a}$

$$
\min _{x \in \mathbb{R}^{p}, r>0} h(D, B n(x, r))=\left(R\left(x_{0}\right)-\rho_{\Omega}\left(x_{0}\right)\right) / 2 .
$$

Поэтому в данном случае можно говорить об эквивалентности задач (1.1) и (1.4). Последняя с геометрической точки зрения является задачей о построении шарового слоя "наименьшей толшины”, содержащего границу выпуклого компакта $D$. Как отмечалось в [2], такая задача или близкие к ней рассматривались в [3]-[7] на плоскости, т.е. при $p=2$, и при произвольном $p$ в [5], [8]-[10], но опять же для евклидовой нормы.

Следует также отметить, что функция $R(x)$ является выпуклой на $\mathbb{R}^{p}$, а функция $\rho_{\Omega}(x)$ - вогнутой на $D$. Поэтому задача (1.4) является задачей выпуклого программирования. Однако для произвольной нормы задачи (1.1) и (1.4) могут быть не эквивалентными. Как показывают примеры, среди шаров наилучшего приближения могут быть такие, центры которых не содержатся в $D$. K тому же решение задачи может быть не единственным. И все же при любой норме $n(\cdot)$, как будет доказано в $\S 3$, задача (1.1) эквивалентна одной задаче выпуклого программирования, которая несколько отличается от (1.4). А именно, имеет место следующая

ТЕОРемА 1.1. Задача (1.1) әквивалентна задаче

$$
\Phi(x) \equiv R(x)+P(x) \rightarrow \min _{x \in \mathbb{R}^{p}}
$$


где $P(x)=\rho_{D}(x)-\rho_{\Omega}(x)$. Причем если пара $\left(x_{0}, r_{0}\right)$ является решением задачи (1.1), то точка $x_{0}$ является решением задачи (1.5) и $r_{0}=$ $\left(R\left(x_{0}\right)-P\left(x_{0}\right)\right) / 2$. И наоборот, если точка $x_{0}$ - решение задачи (1.5), то пара $\left(x_{0}, r_{0}\right)$, где $r_{0}=\left(R\left(x_{0}\right)-P\left(x_{0}\right)\right) / 2$, является решением задачи (1.1). При этом

$$
\min _{x \in \mathbb{R}^{p}, r>0} h(D, B n(x, r))=\left(R\left(x_{0}\right)+P\left(x_{0}\right)\right) / 2 .
$$

В $\S 3$ будет показано, что функция $P(x)$ является вьпуклой на $\mathbb{R}^{p}$. Поэтому задача (1.5) является задачей выпуклого программирования, а теорема 1.1 дает возможность использовать для исследования задачи (1.1) средства выпуклого анализа. В $\S 4$ получены необходимые и достаточные условия решения задачи и некоторые его свойства. В частности, доказано, что центр хотя бы одного из шаров наилучшего приближения содержится в $D$; приведены условия, при которых все множество центров шаров наилучшего приближения содержится в $D$; получено условие единственности решения задачи.

\section{§ 2. Вспомогательные функции и их свойства}

Поскольку норма $n(x)$ является выпуклой и конечной на $\mathbb{R}^{p}$ функцией, то она непрерывна и дифференцируема в любой точке $x \in \mathbb{R}^{p}$ по любому направлению $g \in \mathbb{R}^{p}$, а ее субдифференциал $\partial n(\cdot)$ как многозначное отображение (м.о.) является полунепрерывным сверху, причем

$$
n^{\prime}(x, g) \equiv \lim _{\alpha \downarrow 0} \alpha^{-1}[n(x+\alpha g)-n(x)]=\max _{v \in \partial n(x)}\langle v, g\rangle
$$

где $\langle\cdot, \cdot\rangle$ - скалярное произведение. Известна формула субдифференциала нормы (cM. [11]):

$$
\begin{gathered}
\partial n\left(0_{p}\right)=\left\{v \in \mathbb{R}^{p}: n^{*}(v) \leqslant 1\right\} \\
\partial n(x)=\left\{v \in \mathbb{R}^{p}: n^{*}(v)=1, n(x)=\langle v, x\rangle\right\}, \quad x \neq 0_{p} .
\end{gathered}
$$

Здесь $n^{*}(\cdot)=\max _{n(v) \leqslant 1}\langle v, \cdot\rangle$, а $0_{p}=(0, \ldots, 0) \in \mathbb{R}^{p}$.

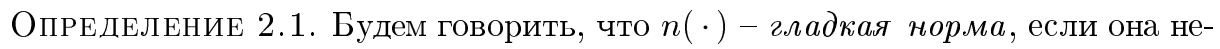
прерывно дифференцируема во всех точках $x \neq 0_{p}$.

В соответствии с понятием строго квазивьпуклой функции (см., например, [12; $\S 4$, п. 6]) дадим следующее определение.

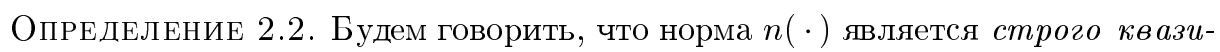
въпуклой, если

$$
n\left(\alpha x_{1}+(1-\alpha) x_{2}\right)<\max \left\{n\left(x_{1}\right) ; n\left(x_{2}\right)\right\} \quad \forall x_{1} \neq x_{2}, \quad \alpha \in(0,1)
$$

Примером строго квазивыпуклой нормы является евклидова и “эллипсоидальная" норма (т.е. шар которой является эллипсоидом), а также любая калибровочная функция [13], порожденная строго выпуклым [14] или сильно выпуклым [15] компактом, симметричным относительно нулевого элемента. Чебышёвская норма 
$n(x)=\max _{i=1, \ldots, p}\left|x^{(i)}\right|$, где $x=\left(x^{(1)}, x^{(2)}, \ldots, x^{(p)}\right) \in \mathbb{R}^{p}$, является примером нормы, не удовлетворяющей определению 2.2 .

Естественно ожидать, что свойства используемой нормы будут отражаться на свойствах решения задачи (1.1). Они же могут обусловливать и выбор средств исследования. Так, известна простая формула, выражающая расстояние между множествами в метрике Хаусдорфа, порождаемой евклидовой нормой, через опорные функции этих множеств (см., например, [14]). Это, видимо, предопределило систематическое использование аппарата опорных функций в работе [2]. Для нас же в дальнейшем изложении важную роль будут играть следующие вспомогательные функции:

$R(x)$ - функция, выражающая радиус наименьшего шара с центром в точке $x$, содержашего множество $D$;

$\rho_{A}(x)$ - функция, выражаюшая расстояние от точки $x$ до множества $A$;

$P(x)=\rho_{D}(x)-\rho_{\Omega}(x)$, где $\Omega=\overline{\mathbb{R}^{p} \backslash D}$.

В этом параграфе мы рассмотрим некоторые свойства этих функций, используя следующие обозначения:

$\bar{A}, \operatorname{int} A, \operatorname{co} A, \partial A$-соответственно замыкание, внутренность, выпуклая оболочка и граница множества $A$;

$$
\begin{gathered}
A+B=\{a+b: a \in A, b \in B\} ; \quad A-B=\{a-b: a \in A, b \in B\} ; \\
K(A)=\left\{v \in \mathbb{R}^{p}: \exists \alpha \geqslant 0, a \in A: v=\alpha a\right\} ; \\
K^{+}=\left\{w \in \mathbb{R}^{p}:\langle v, w\rangle \geqslant 0 \forall v \in K\right\} ;
\end{gathered}
$$

$K(x, A)$ - конус возможных направлений множества $A$ в точке $x$, т.е. $K(x, A)=$ $\bar{\gamma}(x, A)$, где $\gamma(x, A)=\left\{g \in \mathbb{R}^{p}: \exists \alpha_{g} \geqslant 0: x+\alpha g \in A, \alpha \in\left(0, \alpha_{0}\right)\right\}$;

$$
\begin{gathered}
Q^{R}(x, D)=\{y \in D: n(x-y)=R(x)\} ; \\
Q^{\rho}(x, A)=\left\{z \in A: n(x-z)=\rho_{A}(x)\right\} ; \\
{\left[x_{1}, x_{2}\right]=\operatorname{co}\left\{x_{1} ; x_{2}\right\} \quad \text { - отрезок, соединяющий точки } x_{1} \text { и } x_{2} ;} \\
\|x\|=\left(\sum_{i=1}^{p}\left(x^{(i)}\right)^{2}\right)^{1 / 2} \text { - евклидова норма элемента } x \in \mathbb{R}^{p} .
\end{gathered}
$$

TЕОРЕМА 2.1. Функиии $R(x)$ и $\rho_{A}(x)$ являются глобально липиичевыми на $\mathbb{R}^{p}$, причем для любъх $x, y \in \mathbb{R}^{p}$ выполняется

$$
\begin{aligned}
\mid R(x)-R(y)) \mid & \leqslant n(x-y), \\
\left|\rho_{A}(x)-\rho_{A}(y)\right| & \leqslant n(x-y) .
\end{aligned}
$$

ДокАЗАТЕЛЬСТво. Применяя неравенство треугольника, для нормы $n(\cdot)$ получаем

$$
\begin{aligned}
|R(x)-R(y)| & =\left|\max _{z \in D} n(x-z)-\max _{z \in D} n(y-z)\right| \\
& \leqslant \max _{z \in D}|n(x-z)-n(y-z)| \leqslant n(x-y) .
\end{aligned}
$$


Неравенство (2.4) получается так же, как и в [16], [17], для случая евклидовой нормы. Глобальная липшицевость данных функций следует из (2.3), (2.4) в силу эквивалентности норм в $\mathbb{R}^{p}$.

Используя известные факты из субдифференциального исчисления выпуклых функций (см., например, [16; теорема 3.6]), а также замкнутость множества $Q^{R}(x, D)$ и полунепрерывность сверху субдифференциала нормы $\partial n(\cdot)$ как м.о., нетрудно доказать, что справедлива

ТЕОРема 2.2. Функиия $R(x)$ является выпуклой на $\mathbb{R}^{p}$, причем ее субдифференциал можсно выразить формулой

$$
\underline{\partial R}(x)=\operatorname{co}\left\{\partial n(x-z): z \in Q^{R}(x, D)\right\} .
$$

ЛЕмма 2.1. Если $n(\cdot)$ - строго квазивыпуклая норма, а әлементы $x_{1} \neq 0_{p}$, $x_{2} \neq 0_{p}$ и $x_{2} \neq \lambda x_{1}$ для любого $\lambda>0$, то

$$
n\left(\alpha x_{1}+(1-\alpha) x_{2}\right)<\alpha n\left(x_{1}\right)+(1-\alpha) n\left(x_{2}\right), \quad \alpha \in(0,1) .
$$

ДокАЗАТЕЛьСтво. Если $n\left(x_{1}\right)=n\left(x_{2}\right)$, то (2.6) следует сразу из $(2.2)$. Пусть $n\left(x_{1}\right) \neq n\left(x_{2}\right)$. Возьмем элемент $x_{3}=\frac{n\left(x_{2}\right)}{n\left(x_{1}\right)} x_{1}$. В силу условия леммы $x_{3} \neq x_{2}$ и в то же время $n\left(x_{3}\right)=n\left(x_{2}\right)$. Поэтому в соответствии с определением 2.2 имеем

$$
n\left(\beta x_{3}+(1-\beta) x_{2}\right)<n\left(x_{2}\right) \quad \forall \beta \in(0,1) .
$$

Возьмем произвольное $\alpha \in(0,1)$ и поставим ему в соответствие числа

$$
\beta_{0}=\frac{\alpha n\left(x_{1}\right)}{\alpha n\left(x_{1}\right)+(1-\alpha) n\left(x_{2}\right)}, \quad \lambda_{0}=\frac{\alpha n\left(x_{1}\right)+(1-\alpha) n\left(x_{2}\right)}{n\left(x_{2}\right)} .
$$

Легко убедиться, что $\beta_{0} \in(0,1)$ и $\alpha x_{1}+(1-\alpha) x_{2}=\lambda_{0}\left(\beta_{0} x_{3}+\left(1-\beta_{0}\right) x_{2}\right)$. Тогда, используя неравенство (2.7), получаем (2.5):

$n\left(\alpha x_{1}+(1-\alpha) x_{2}\right)=\lambda_{0} n\left(\beta_{0} x_{3}+\left(1-\beta_{0}\right) x_{2}\right)<\lambda_{0} n\left(x_{2}\right)=\alpha n\left(x_{1}\right)+(1-\alpha) n\left(x_{2}\right)$.

ЛЕмма 2.2. Если $n(\cdot)$ - строго квазивыпуклая норма, то равенство

$$
n\left(x_{2}\right)=n\left(x_{1}\right)+n\left(x_{1}-x_{2}\right)
$$

для $x_{1} \neq 0_{p}$ выполняется тогда и только тогда, когда существует $\lambda \geqslant 1$ maкое, чmo $x_{2}=\lambda x_{1}$.

ДокАЗАтЕльство. Достаточность очевидна. Докажем необходимость. Пусть имеет место равенство $(2.8)$ и $x_{1} \neq 0_{p}$. Если предположить противное утверждению леммы 2.2, то, применяя лемму 2.1, получим неравенство (2.6). Из него и (2.8) следует неравенство

$$
n\left(x_{2}\right)-n\left(\alpha x_{1}+(1-\alpha) x_{2}\right)>\alpha n\left(x_{1}-x_{2}\right), \quad \alpha \in(0,1),
$$

противоречашее неравенству треугольника для нормы $n(\cdot)$. 
TЕОРема 2.3. Если точки $x_{1}$ и $x_{2}$ таковы, что

$$
R\left(x_{2}\right)=R\left(x_{1}\right)+n\left(x_{1}-x_{2}\right)
$$

то для всех $\alpha \in[0,1]$ выполняется равенство

$$
R\left(\alpha x_{1}+(1-\alpha) x_{2}\right)=\alpha R\left(x_{1}\right)+(1-\alpha) R\left(x_{2}\right)
$$

Если же $n(\cdot)$ - строго квазивыпуклая норма и для точек $x_{1}$ и $x_{2}$ выполняются неравенства

$$
R\left(x_{1}\right) \leqslant R\left(x_{2}\right)<R\left(x_{1}\right)+n\left(x_{1}-x_{2}\right),
$$

то имеет место строгое неравенство

$$
R\left(\alpha x_{1}+(1-\alpha) x_{2}\right)<\alpha R\left(x_{1}\right)+(1-\alpha) R\left(x_{2}\right), \quad \alpha \in(0,1)
$$

ДокАзАтЕЛьство. а) Пусть вьполняется (2.9), но найдется $\alpha_{0} \in(0,1)$ такое, что выполняется неравенство

$$
R\left(\alpha_{0} x_{1}+\left(1-\alpha_{0}\right) x_{2}\right)<\alpha_{0} R\left(x_{1}\right)+\left(1-\alpha_{0}\right) R\left(x_{2}\right)=R\left(x_{2}\right)-\alpha_{0} n\left(x_{1}-x_{2}\right)
$$

Однако, применяя теорему 2.1, получаем противоречащее ему неравенство

$$
R\left(x_{2}\right)-R\left(\alpha_{0} x_{1}+\left(1-\alpha_{0}\right) x_{2}\right) \leqslant \alpha_{0} n\left(x_{1}-x_{2}\right)
$$

что и доказывает первую часть утверждения теоремы.

б) Пусть теперь $n(\cdot)$ - строго квазивьпуклая норма и выполняется неравенство (2.11). Если предположить, что хотя бы для одного значения $\alpha_{0} \in(0,1)$ неравенство (2.12) не выполняется, то из выпуклости функции $R(x)$ легко следует, что равенство (2.10) выполняется для всех $\alpha \in[0,1]$ или

$$
R\left(x_{1}+(1-\alpha)\left(x_{2}-x_{1}\right)\right)-R\left(x_{1}\right)=(1-\alpha)\left(R\left(x_{2}\right)-R\left(x_{1}\right)\right)
$$

Поделив обе части равенства на $\beta=1-\alpha$ и перейдя к пределу при $\beta \downarrow 0$, имеем

$$
R^{\prime}\left(x_{1}, x_{2}-x_{1}\right) \equiv \lim _{\beta \downarrow 0} \beta^{-1}\left[R\left(x_{1}+\beta\left(x_{2}-x_{1}\right)\right)-R\left(x_{1}\right)\right]=R\left(x_{2}\right)-R\left(x_{1}\right) .
$$

Полунепрерывность сверху м.о. $\partial n(\cdot): \mathbb{R}^{p} \rightarrow 2^{\mathbb{R}^{p}}$, ограниченность $\partial n(x)($ см. $(2.1))$ и замкнутость множества $Q^{R}(x, D)$ влекут компактность множества $\{\partial n(x-z)$ : $\left.z \in Q^{R}(x, D)\right\}$. Тогда, применяя теорему 2.2 , получаем, что найдется элемент $z_{0} \in Q^{R}\left(x_{1}, D\right)$ такой, что

$$
\begin{aligned}
R^{\prime}\left(x_{1}, x_{2}-x_{1}\right) & =\max _{v \in \underline{\partial R}\left(x_{1}\right)}\left\langle v, x_{2}-x_{1}\right\rangle=\max _{v \in\left\{\partial n\left(x_{1}-z\right): z \in Q^{R}\left(x_{1}, D\right)\right\}}\left\langle v, x_{2}-x_{1}\right\rangle \\
& =\max _{v \in \partial n\left(x_{1}-z_{0}\right)}\left\langle v, x_{2}-x_{1}\right\rangle=n^{\prime}\left(x_{1}-z_{0}, x_{2}-x_{1}\right) .
\end{aligned}
$$


Поскольку $z_{0} \in D$ и $R\left(x_{1}\right)=n\left(x_{1}-z_{0}\right)$, то, используя правую часть неравенства (2.11), имеем

$$
n\left(x_{2}-z_{0}\right) \leqslant R\left(x_{2}\right)<n\left(x_{1}-z_{0}\right)+n\left(x_{1}-x_{2}\right)
$$

Применяя теперь последовательно леммы 2.2 и 2.1 , получаем неравенство

$$
n\left(\alpha x_{1}+(1-\alpha) x_{2}-z_{0}\right)<\alpha n\left(x_{1}-z_{0}\right)+(1-\alpha) n\left(x_{2}-z_{0}\right), \quad \alpha \in(0,1) .
$$

Таким образом, выпуклая функция $f(x)=n\left(x-z_{0}\right)$ не является аффинной на отрезке $\left[x_{1}, x_{2}\right]$ и, следовательно,

$n\left(x_{2}-z_{0}\right)=f\left(x_{2}\right)>f\left(x_{1}\right)+f^{\prime}\left(x_{1}, x_{2}-x_{1}\right)=n\left(x_{1}-z_{0}\right)+n^{\prime}\left(x_{1}-z_{0}, x_{2}-x_{1}\right)$.

Отсюда, используя (2.14) и (2.13), получаем

$$
\begin{aligned}
R\left(x_{2}\right) & \geqslant n\left(x_{2}-z_{0}\right)>R\left(x_{1}\right)+n^{\prime}\left(x_{1}-z_{0}, x_{2}-x_{1}\right) \\
& =R\left(x_{1}\right)+R^{\prime}\left(x_{1}, x_{2}-x_{1}\right)=R\left(x_{2}\right)
\end{aligned}
$$

- противоречие.

В [18] доказаны следующие два факта.

ТЕОРЕМА 2.4. Функиия $\rho_{D}(x)$ является выпуклой на $\mathbb{R}^{p}$, причем ее субдифференциал можсно выразить формулой

$$
\underline{\partial \rho}_{D}(x)=\partial n(x-z) \cap-K^{+}(z, D) \quad \forall z \in Q^{\rho}(x, D) .
$$

ТЕОРЕМА 2.5. Функция $\rho_{\Omega}(x)$ является вогнутой на $D$, причем ее условный супердифференциал можсно выразить формулой

$$
\overline{\rho_{\Omega}}(x)=\operatorname{co}\left\{\partial n(x-z) \cap K^{+}(z, D): z \in Q^{\rho}(x, \Omega)\right\}, \quad x \in \operatorname{int} D
$$

$B$ граничных точках множества $D$ функция $\rho_{\Omega}(x)$ дифференцируема по любому направлению $g \in \mathbb{R}^{p}$, причем

$$
\rho_{\Omega}^{\prime}(x, g) \equiv \lim _{\alpha \downarrow 0} \alpha^{-1}\left[\rho_{\Omega}(x+\alpha g)-\rho_{\Omega}(x)\right]=\max \left\{0 ; \min _{\substack{w \in K^{+}(x, D) \\ n^{*}(w)=1}}\langle w, g\rangle\right\}
$$

ЗАмечаниЕ 2.1. Нетрудно показать (см. [18]), что $K^{+}(z, D) \subset K(\partial n(x-z))$ для всех $z \in Q^{\rho}(x, D)$ при $x \in D$. Поэтому справедлива также формула

$$
\overline{\rho_{\Omega}}(x)=\operatorname{co}\left\{w \in K^{+}(z, D): z \in Q^{\rho}(x, \Omega), n^{*}(w)=1\right\}, \quad x \in \operatorname{int} D
$$

Далеев зависимости от обстоятельств будем использовать обе формулы для $\overline{\rho \Omega}(x)$. 
ЛЕмма 2.3. Пусть функиии $\psi_{1}(t)$ и $\psi_{2}(t)$ определень, выпукльи и конечны соответственно на отрезках $[a, b] u[b, c]$, причем $a<b<c u \psi_{1}(b)=\psi_{2}(b)$. Если существует $\alpha \in(0,1)$ такое, что для функиии

$$
\psi(t)= \begin{cases}\psi_{1}(t), & t \in[a, b] \\ \psi_{2}(t), & t \in[b, c]\end{cases}
$$

въполняется неравенство

$$
\psi(\alpha a+(1-\alpha) c)>\alpha \psi(a)+(1-\alpha) \psi(c)
$$

то для точки $b$, представимой в виде

$$
b=\beta a+(1-\beta) c, \quad \beta \in(0,1)
$$

выполняется неравенство

$$
\psi(b)>\beta \psi(a)+(1-\beta) \psi(c)
$$

ДокАЗАТЕЛьСтво. Предположим противное, т.е.

$$
\psi(b) \leqslant \beta \psi(a)+(1-\beta) \psi(c)
$$

Рассмотрим случай, когда $\alpha \in(0, \beta)$. Поскольку $\psi(t)$ выпукла на отрезке $[b, c]$, то для $\gamma=\alpha / \beta$ можно записать неравенство

$$
\psi(\gamma b+(1-\gamma) c) \leqslant \gamma \psi(b)+(1-\gamma) \psi(c)
$$

или, подставляя сюда (2.20) и используя (2.22), получим

$$
\psi(\alpha a+(1-\alpha) c) \leqslant \alpha \psi(a)+(1-\alpha) \psi(c)
$$

что противоречит (2.19). Аналогично, используя вьпуклость $\psi(t)$ на отрезке $[a, b]$, рассматривается случай $\alpha \in(\beta, 1)$.

ТЕОРЕМА 2.6. Функция $P(x)$ является выпуклой и конечной на $\mathbb{R}^{p}$, причем ее субдифференциал можно записать в виде

$$
\underline{\partial P}(x)= \begin{cases}\partial n(x-z) \cap-K^{+}(z, D) \quad \forall z \in Q^{\rho}(x, D), & \text { если } x \notin D, \\ \operatorname{co}\left\{v \in-K^{+}(z, D): n^{*}(v)=1, z \in Q^{\rho}(x, \Omega)\right\}, & \text { если } x \in D .\end{cases}
$$


ДокАЗАТЕльство. Предположим, что функция $P(x)$ не является выпуклой на $\mathbb{R}^{p}$, т.е. найдутся точки $x_{1}, x_{2}$ и число $\alpha_{0} \in(0,1)$ такие, что

$$
P\left(\alpha_{0} x_{1}+\left(1-\alpha_{0}\right) x_{2}\right)>\alpha_{0} P\left(x_{1}\right)+\left(1-\alpha_{0}\right) P\left(x_{2}\right) .
$$

Как следует из теорем 2.2 и 2.5 , а также определения функции $P(x)$, нетривиальньми для рассмотрения являются только случаи, когда $x_{1} \in$ int $D$ и $x_{2} \notin D$ или $x_{1}, x_{2} \notin D$, но $\left[x_{1}, x_{2}\right] \cap$ int $D \notin \varnothing$.

a) Пусть $x_{1} \in \operatorname{int} D, x_{2} \notin D$. Обозначим через $x_{0}$ граничную точку множества $D$, принадлежашую отрезку $\left[x_{1}, x_{2}\right]$. Функция $P(x)$ выпукла на отрезке $\left[x_{1}, x_{0}\right]$ и на отрезке $\left[x_{0}, x_{2}\right]$ по теоремам 2.5 и 2.2. Тогда по лемме 2.3 из неравенства $(2.24)$ для точки $x_{0}=\beta x_{1}+(1-\beta) x_{2}$, где $\beta \in(0,1)$, имеем

$$
P\left(x_{0}\right)>\beta P\left(x_{1}\right)+(1-\beta) P\left(x_{2}\right)
$$

Покажем, что из (2.25) для $g=\frac{x_{2}-x_{1}}{\left\|x_{2}-x_{1}\right\|}$ выполняется неравенство

$$
P^{\prime}\left(x_{0}, g\right)<-P^{\prime}\left(x_{0},-g\right) \text {. }
$$

Действительно, непосредственно из (2.25) получаем

$$
(1-\beta)\left(P\left(x_{2}\right)-P\left(x_{0}\right)\right)<\beta\left(P\left(x_{0}\right)-P\left(x_{1}\right)\right) .
$$

А так как $\frac{\beta}{1-\beta}=\frac{\left\|x_{2}-x_{0}\right\|}{\left\|x_{1}-x_{0}\right\|}$, то имеем

$$
\frac{P\left(x_{2}\right)-P\left(x_{0}\right)}{\left\|x_{2}-x_{0}\right\|}<\frac{P\left(x_{0}\right)-P\left(x_{1}\right)}{\left\|x_{1}-x_{0}\right\|} .
$$

Из выпуклости функции $P(x)$ на отрезках $\left[x_{0}, x_{2}\right]$ и $\left[x_{1}, x_{0}\right]$ следуют соответствующие неравенства

$$
P^{\prime}\left(x_{0}, g\right) \leqslant \frac{P\left(x_{2}\right)-P\left(x_{0}\right)}{\left\|x_{2}-x_{0}\right\|}, \quad P^{\prime}\left(x_{0},-g\right) \leqslant \frac{P\left(x_{1}\right)-P\left(x_{0}\right)}{\left\|x_{1}-x_{0}\right\|} .
$$

Из (2.28), (2.29) получаем (2.26).

А теперь покажем, что на самом деле выполняется

$$
P^{\prime}\left(x_{0}, g\right) \geqslant-P^{\prime}\left(x_{0},-g\right)
$$

Действительно, поскольку $x_{0}$ - граничная точка выпуклого множества $D$, то в соответствии с известным фактом из выпуклого анализа (см., например, [12], [13]) существует опорная гиперплоскость $\pi$, которая содержит точку $x_{0}$ и отсекает множество $D$ в одно из полупространств, на которые она разбивает все пространство $\mathbb{R}^{p}$. Тогда из определения функции $P(x)$, свойств опорной гиперплоскости $\pi$ и выбора точек $x_{1}$ и $x_{2}$ следует, что при достаточно малых $\alpha \geqslant 0$

$$
\begin{gathered}
\rho_{\pi}\left(x_{0}+\alpha g\right)=\rho_{\pi}\left(x_{0}-\alpha g\right), \quad P\left(x_{0}+\alpha g\right)=\rho_{D}\left(x_{0}+\alpha g\right) \geqslant \rho_{\pi}\left(x_{0}+\alpha g\right), \\
P\left(x_{0}-\alpha g\right)=-\rho_{\Omega}\left(x_{0}-\alpha g\right) \geqslant-\rho_{\pi}\left(x_{0}-\alpha g\right), \quad \rho_{\pi}\left(x_{0}\right)=P\left(x_{0}\right)=0 .
\end{gathered}
$$


Отсюда легко следует

$$
\begin{gathered}
P^{\prime}\left(x_{0}, g\right) \geqslant \rho_{\pi}^{\prime}\left(x_{0}, g\right), \\
P^{\prime}\left(x_{0},-g\right) \geqslant-\rho_{\pi}^{\prime}\left(x_{0},-g\right), \\
\rho_{\pi}^{\prime}\left(x_{0}, g\right)=\rho_{\pi}^{\prime}\left(x_{0},-g\right) .
\end{gathered}
$$

Из (2.31) получаем (2.30).

Полученное противоречие (2.30) с (2.26) говорит о том, что наше предположение $(2.24)$ в рассматриваемом случае было неверно.

б) Пусть теперь $x_{1}, x_{2} \notin D$, но $\left[x_{1}, x_{2}\right] \cap$ int $D \neq \varnothing$. Функция $P(x)$ является непрерывной. Поэтому из ее определения следует существование точки $x_{0} \in$ $\left[x_{1}, x_{2}\right] \cap \operatorname{int} D$, в которой

$$
P\left(x_{0}\right)=\min _{x \in\left[x_{1}, x_{2}\right]} P(x)<0<\min \left\{P\left(x_{1}\right) ; P\left(x_{2}\right)\right\}
$$

Как было доказано в п. а), функция $P(x)$ выпукла на отрезках $\left[x_{1}, x_{0}\right]$ и $\left[x_{0}, x_{2}\right]$. Из предположения (2.24) по лемме 2.3 для точки $x_{0}=\beta x_{1}+(1-\beta) x_{2}$, где $\beta \in(0,1)$, следует неравенство $P\left(x_{0}\right)>\beta P\left(x_{1}\right)+(1-\beta) P\left(x_{2}\right)$, которое противоречит (2.32).

в) Нам осталось доказать формулу (2.23). Для случаев, когда $x \notin D$ или $x \in \operatorname{int} D$, она следует непосредственно из теорем 2.4 и 2.5 . Поэтому нам осталось доказать ее справедливость для случая, когда $x$ - граничная точка множества $D$. Используя теоремы $2.4,2.5$ и формулу (2.1), получаем

$$
\begin{aligned}
& P^{\prime}(x, g)=\rho_{D}^{\prime}(x, g)-\rho_{\Omega}^{\prime}(x, g) \\
& =\max _{v \in \partial n(0) \cap-K^{+}(x, D)}\langle v, g\rangle-\max \left\{0 ; \min _{\substack{w \in K^{+}(x, D) \\
n^{*}(v)=1}}\langle w, g\rangle\right\} \\
& =\max \left\{0 ; \max _{\substack{v \in-K^{+}(x, D) \\
n^{*}(v)=1}}\langle v, g\rangle\right\}+\min \left\{0 ; \max _{\substack{v \in-K^{+}(x, D) \\
n^{*}(v)=1}}\langle v, g\rangle\right\} \\
& =\max _{\substack{v \in-K^{+}(x, D) \\
n^{*}(v)=1}}\langle v, g\rangle=\max _{\operatorname{co}\left\{v \in-K^{+}(x, D): n^{*}(v)=1\right\}}\langle v, g\rangle \quad \forall g \in \mathbb{R}^{p} .
\end{aligned}
$$

С другой стороны, для выпуклой конечной функции $P(x)$ имеет место формула производной по направлениям, выраженная с помощью ее субдифференциала $\underline{\partial P}(x)$, который является выпуклым компактом

$$
P^{\prime}(x, g)=\max _{v \in \underline{\partial P}(x)}\langle v, g\rangle \quad \forall g \in \mathbb{R}^{p}
$$

Из (2.33), (2.34) следует $\underline{\partial P}(x)=\operatorname{co}\left\{v \in-K^{+}(x, D): n^{*}(v)=1\right\}$, что соответствует формуле (2.23) для случая $x \in \partial D$. 


\section{§ 3. Редукция к задаче выпуклого программирования}

Докажем ряд вспомогательных фактов.

ЛЕмма 3.1. Справедлива формула

$$
\rho(D, B n(x, r))= \begin{cases}0, & \text { eсли } r \geqslant R(x), \\ R(x)-r, & \text { если } 0 \leqslant r<R(x) .\end{cases}
$$

ДокАЗАтЕЛЬСтво. 1) Если $r \geqslant R(x)$, то $D \subset B n(x, r)$ и, следовательно, $\rho(D, B n(x, r))=0$.

2) Пусть $0 \leqslant r<R(x)$. Возьмем точку $y_{0} \in Q^{R}(x, D)$, т.е. $y_{0} \in D$ и $n\left(x-y_{0}\right)=R(x)$, и точку $x_{0} \in\left[x, y_{0}\right]$ такую, что $n\left(x-x_{0}\right)=r$. Ясно, что $x_{0} \in B n(x, r)$ и тогда, поскольку $D \subset B n(x, R(x))$, получаем

$$
R(x)-r=n\left(y_{0}-x_{0}\right) \leqslant \rho(D, B n(x, r)) \leqslant \rho(B n(x, R(x)), B n(x, r))=R(x)-r .
$$

ЛЕмма 3.2. Если $x \in D$, то справедлива формула

$$
\rho(B n(x, r), D)= \begin{cases}0, & \text { если } 0 \leqslant r \leqslant \rho_{\Omega}(x), \\ r-\rho_{\Omega}(x), & \text { если } r>\rho_{\Omega}(x) .\end{cases}
$$

ДокАЗАТЕЛьСтво. 1) Если $0 \leqslant r \leqslant \rho_{\Omega}(x)$, то $B n(x, r) \subset D$ и, следовательно,

$$
\rho(B n(x, r), D)=0
$$

2) Пусть $r>\rho_{\Omega}(x)$. Поскольку $B n\left(x, \rho_{\Omega}(x)\right) \subset D$, то

$$
\rho(B n(x, r), D) \leqslant \rho\left(B n(x, r), B n\left(x, \rho_{\Omega}(x)\right)\right)=r-\rho_{\Omega}(x) .
$$

Возьмем точку $y_{0} \in Q^{\rho}(x, \Omega)$, т.е.

$$
y_{0} \in \Omega, \quad \rho_{\Omega}(x)=n\left(x-y_{0}\right)
$$

и точку $z_{0}$ такую, что $n\left(x-z_{0}\right)=r$ и $y_{0} \in\left[x, z_{0}\right]$. Докажем, что

$$
\rho_{D}\left(z_{0}\right) \equiv \min _{y \in D} n\left(z_{0}-y\right)=r-\rho_{\Omega}(x) .
$$

В соответствии с выбором точек $y_{0}$ и $z_{0}$ выполняется

$$
n\left(z_{0}-y_{0}\right)=r-\rho_{\Omega}(x)
$$

Покажем, что

$$
\rho_{D}\left(z_{0}\right)=n\left(z_{0}-y_{0}\right) .
$$

Действительно, $B n\left(x, \rho_{\Omega}(x)\right) \subset D$ и, следовательно,

$$
K\left(y_{0}, B n\left(x, \rho_{\Omega}(x)\right)\right) \subset K\left(y_{0}, D\right)
$$


Так как $\left.B n\left(x, \rho_{\Omega}(x)\right)=\left\{y \in \mathbb{R}^{p}: n(y-x)-\rho_{\Omega}(x) \leqslant 0\right)\right\}$, то, учитывая (3.3) и применяя известную формулу для конуса возможных направлений лебегова множества выпуклой функции через ее субдифференциал (см., например, [12; §12, п. 2]), имеем

$$
K\left(y_{0}, B n\left(x, \rho_{\Omega}(x)\right)\right)=-K^{+}\left(\partial n\left(y_{0}-x\right)\right) .
$$

Поэтому из (3.7) получаем $K^{+}\left(y_{0}, D\right) \subset K\left(\partial n\left(x-y_{0}\right)\right)$ и, следовательно, $\partial n\left(x-y_{0}\right) \cap K^{+}\left(y_{0}, D\right) \neq \varnothing$. Поскольку из положительной однородности нормы следует $\partial n\left(x-y_{0}\right)=\partial n\left(y_{0}-z_{0}\right)$, то и

$$
\partial n\left(y_{0}-z_{0}\right) \cap K^{+}\left(y_{0}, D\right) \neq \varnothing .
$$

Это соотношение в соответствии с известньм фактом из выпуклого анализа (см. [16; теорема 2.1]) означает, что

$$
n\left(z_{0}-y_{0}\right)=\min _{y \in D} n\left(z_{0}-y\right)
$$

т.е. вьполняется (3.6).

Теперь (3.4) следует из (3.5), (3.6). Поскольку $z_{0} \in B n(x, r)$, то из (3.4) имеем

$$
r-\rho_{\Omega}(x)=\rho_{D}\left(z_{0}\right) \leqslant \rho(B n(x, r), D) .
$$

Из (3.2) и (3.8) получаем требуемое.

Лемма 3.3. Если $x \notin D, m o$

$$
\rho(B n(x, r), D)=r+\rho_{D}(x) .
$$

ДокАЗАТЕЛЬСТво. Возьмем $y_{0} \in Q^{\rho}(x, D)$, т.е.

$$
y_{0} \in D, \quad \rho_{D}(x) \equiv \min _{y \in D} n(x-y)=n\left(x-y_{0}\right),
$$

и точку $z_{0}=x+r\left(n\left(x-y_{0}\right)\right)^{-1}\left(x-y_{0}\right)$. Ясно, что $x \in\left[y_{0}, z_{0}\right]$ и $n\left(x-z_{0}\right)=r$, т.е. $z_{0} \in B n(x, r)$. В соответствии с [16; теорема 2.1] (3.10) эквивалентно выполнению соотношения

$$
\partial n\left(y_{0}-x\right) \cap K^{+}\left(y_{0}, D\right) \neq \varnothing .
$$

А поскольку из положительной однородности нормы следует

$$
\partial n\left(y_{0}-x\right)=\partial n\left(y_{0}-z_{0}\right)
$$

то и

$$
\partial n\left(y_{0}-z_{0}\right) \cap K^{+}\left(y_{0}, D\right) \neq \varnothing .
$$

Это означает, что

$$
\rho_{D}\left(z_{0}\right) \equiv \min _{y \in D} n\left(z_{0}-y\right)=n\left(y_{0}-z_{0}\right)
$$


А так как $n\left(y_{0}-z_{0}\right)=\rho_{D}(x)+r$, то получаем

$$
\rho_{D}\left(z_{0}\right)=\rho_{D}(x)+r .
$$

Обозначим через $G\left(z_{0}\right)=\left\{y \in \mathbb{R}^{p}: \rho_{D}(y) \leqslant \rho_{D}\left(z_{0}\right)\right\}$. Нетрудно показать (см.[19]), что $G\left(z_{0}\right)=D+B_{n}\left(0, \rho_{D}\left(z_{0}\right)\right)$ и, следовательно, учитывая, что $y_{0} \in D$, получаем включение

$$
B n\left(y_{0}, \rho_{D}\left(z_{0}\right)\right)=y_{0}+B n\left(0, \rho_{D}\left(z_{0}\right)\right) \subset G\left(x_{0}\right) .
$$

Если $y \in B n(x, r)$, то, учитывая (3.10), (3.11), имеем

$$
n\left(y_{0}-y\right) \leqslant n\left(y_{0}-x\right)+n(x-y) \leqslant \rho_{D}(x)+r=\rho_{D}\left(z_{0}\right),
$$

т.е. выполняется включение

$$
B n(x, r) \subset B n\left(y_{0}, \rho_{D}\left(z_{0}\right)\right) .
$$

Из (3.12), (3.13) следует $B n(x, r) \subset G\left(z_{0}\right)$ и, значит,

$$
\rho(B n(x, r), D)=\max _{y \in B n(x, r)} \rho_{D}(y) \leqslant \max _{y \in G\left(x_{0}\right)} \rho_{D}(y)=\rho_{D}\left(z_{0}\right) .
$$

Но так как $z_{0} \in B n(x, r)$, то

$$
\max _{y \in B n(x, r)} \rho_{D}(y) \geqslant \rho_{D}\left(z_{0}\right) .
$$

Теперь из (3.14), (3.15) и (3.11) получаем (3.9).

Лемма 3.4. Имеет место равенство

$$
\min _{r \geqslant 0} h(D, B n(x, r))=h\left(D, B n\left(x, r_{0}\right)\right)=(R(x)+P(x)) / 2,
$$

əде $r_{0}=(R(x)-P(x)) / 2$.

ДокАЗАТЕЛЬСтво. 1) Рассмотрим случай $x \in D$. Из лемм 3.1 и 3.2 , учитьвая неравенство $\rho_{\Omega}(x) \leqslant R(x)$, получаем

$$
h(D, B n(x, r))= \begin{cases}R(x)-r, & \text { если } 0 \leqslant r \leqslant \rho_{\Omega}(x), \\ r-\rho_{\Omega}(x), & \text { если } r \geqslant R(x), \\ \max \left\{R(x)-r ; r-\rho_{\Omega}(x)\right\}, & \text { если } \rho_{\Omega}(x) \leqslant r \leqslant R(x) .\end{cases}
$$

Нетрудно убедиться, что

$$
\begin{gathered}
\min _{r \geqslant 0} \max \left\{R(x)-r ; r-\rho_{\Omega}(x)\right\}=\max \left\{R(x)-r_{0} ; r_{0}-\rho_{\Omega}(x)\right\}=\left(R(x)-\rho_{\Omega}(x)\right) / 2 \\
\text { для } r_{0}=\left(R(x)+\rho_{\Omega}(x)\right) / 2 . \text { Поэтому из }(3.17),(3.18) \text { имеем } \\
\qquad \min _{r \geqslant 0} h(D, B n(x, r))=h\left(D, B n\left(x, r_{0}\right)\right)=\left(R(x)-\rho_{\Omega}(x)\right) / 2 .
\end{gathered}
$$

2) Пусть теперь $x \notin D$. Из лемм 3.1 и 3.3 следует

$$
h(D, B n(x, r))=\max \left\{R(x)-r ; r+\rho_{D}(x)\right\} .
$$

Отсюда нетрудно получить

$$
\min _{r \geqslant 0} h(D, B n(x, r))=h\left(D, B n\left(x, r_{0}\right)=\left(R(x)+\rho_{D}(x)\right) / 2,\right.
$$

где $r_{0}=\left(R(x)-\rho_{D}(x)\right) / 2$.

Из (3.19), (3.20) получаем (3.16).

Следствием леммы 3.4 является теорема 1.1. При этом, как следует из теорем 2.2 и 2.6, задача (1.5) является задачей выпуклого программирования. 


\section{§4. Некоторые свойства решения}

Сушествование решения задачи (1.5), а следовательно, и задачи (1.1) легко вытекает из ограниченности и замкнутости лебегова множества $\left\{x \in \mathbb{R}^{p}: R(x)+\right.$ $P(x) \leqslant R(y)+P(y)\}$ и непрерывности функции $\Phi(x)=R(x)+P(x)$. Наличие формул субдифференциалов выпуклых функций $R(x)$ и $P(x)$ позволяет получить необходимое и достаточное условие решения задачи (1.1).

Теорема 4.1. Для того чтобы пара $\left(x_{0}, r_{0}\right)$ была решением задачи (1.1), необходимо и достаточно, чтобъ

$$
0_{p} \in \underline{\partial \Phi}\left(x_{0}\right) \equiv \underline{\partial R}\left(x_{0}\right)+\underline{\partial P}\left(x_{0}\right),
$$

где $\underline{\partial R}\left(x_{0}\right)$ и $\underline{\partial P}\left(x_{0}\right)$ определяются формулами (2.5) и (2.23) соответственно, $a r_{0}=\left(R\left(x_{0}\right)-P\left(x_{0}\right)\right) / 2$.

ДокАЗАТЕЛЬСтво. Как следует из теорем 2.2 и 2.6 , функция $\Phi(x)$ является вьпуклой и конечной на $\mathbb{R}^{p}$. Ее субдифференциал $\underline{\partial \Phi}(x)$ по теореме Моро-Рокафеллара (см. [16; теорема 1.10]) есть алгебраическая сумма $\underline{\partial R}(x)$ и $\underline{\partial P}(x)$. Как известно из выпуклого анализа (см., например, [16; лемма 2.1]), необходимым и достаточным условием решения задачи (1.5) является выполнение включения $0_{p} \in$ $\underline{\partial \Phi}\left(x_{0}\right)$. Теперь осталось применить теорему 1.1 .

ЗАМЕЧАНИЕ 4.1. Из теоремы 1.1 следует, что

$$
X(D)=\left\{y \in \mathbb{R}^{p}: \Phi(y)=\min _{x \in \mathbb{R}^{p}} \Phi(x)\right\}
$$

есть множество всех центров шаров наилучшего приближения для выпуклого компакта $D$ и в соответствии с теоремой 4.1

$$
x_{0} \in X(D) \Leftrightarrow 0_{p} \in \underline{\partial R}\left(x_{0}\right)+\underline{\partial P}\left(x_{0}\right) .
$$

СлЕДСТВИЕ 4.1. Если выпуклый компакт $D$ является центрально симметричным относительно точки $x_{0}$, то $x_{0} \in X(D)$.

ДокАЗАтЕльство. Легко видеть, что $x_{0} \in D$, а множества $Q^{R}\left(x_{0}, D\right)$ и $Q^{\rho}\left(x_{0}, \Omega\right)$ являются центрально симметричными относительно $x_{0}$. Это обстоятельство, как следует из формул (2.1), (2.5), и (2.23), влечет выполнение включений

$$
0_{p} \in \underline{\partial R}\left(x_{0}\right), \quad 0_{p} \in \underline{\partial P}\left(x_{0}\right) .
$$

Поэтому имеет место и (4.1).

ЗАмечАниЕ 4.2. Приведенный далее пример 4.3 показывает, что даже если $D$ обладает центральной симметрией, то множество $X(D)$ может быть не одноточечньгм.

СлЕДСТвИЕ 4.2. Если $D=\left[x_{1}, x_{2}\right]$ - отрезок, соединяющий точки $x_{1}$ и $x_{2}$, то шар наилучшего приближения в задаче (1.1) является единственным. При этом его чентр есть точка $x_{0}=\left(x_{1}+x_{2}\right) / 2$, а радиус $r_{0}=n\left(x_{1}-x_{2}\right) / 4$. 
ДокАЗАТЕЛЬСтво. Очевидно, точка $x_{0}$ является точкой минимума функции $R(x)$ на $\mathbb{R}^{p}$, причем единственной из точек самого отрезка $\left[x_{1}, x_{2}\right]$. Поскольку $P(x)=0$ для $x \in\left[x_{1}, x_{2}\right]$, то $x_{0}$ - единственная точка минимума и функции $\Phi(x)$ на отрезке. Кроме того, в соответствии со следствием $4.1 \Phi\left(x_{0}\right)=\min _{x \in \mathbb{R}^{p}} \Phi(x)$. Теперь осталось заметить, что для точек $x \notin D$ выполняется $P(x)=\rho_{D}(x)>0$ и, значит,

$$
\Phi(x)=R(x)+P(x)>R\left(x_{0}\right)=\Phi\left(x_{0}\right)=\min _{x \in \mathbb{R}^{p}} \Phi(x) .
$$

В соответствии с теоремой 1.1 подсчитываем радиус шара наилучшего приближения

$$
r_{0}=\frac{R\left(x_{0}\right)-P\left(x_{0}\right)}{2}=\frac{R\left(x_{0}\right)}{2}=\frac{n\left(x_{1}-x_{2}\right)}{4} .
$$

Теорема 4.2. Виполняется соотношение

$$
X(D) \cap D \neq \varnothing
$$

причем если $x_{0} \in X(D)$ и $x_{0} \notin D$, mo

$$
\operatorname{co}\left\{x_{0}, Q^{\rho}\left(x_{0}, D\right)\right\} \subset X(D)
$$

ДокАЗАТЕЛЬСтво. Пусть $x_{0} \in X(D)$ и $x_{0} \notin D$. Возьмем любую точку $z \in$ $Q^{\rho}\left(x_{0}, D\right)$. Тогда $P\left(x_{0}\right)=\rho_{D}\left(x_{0}\right)=n\left(x_{0}-z\right), P(z)=0$ и, следовательно,

$$
R\left(x_{0}\right)+n\left(x_{0}-z\right)=R\left(x_{0}\right)+P\left(x_{0}\right)=\Phi\left(x_{0}\right) \leqslant \Phi(z)=R(z) .
$$

С другой стороны, по теореме 2.1 выполняется

$$
R(z)-R\left(x_{0}\right) \leqslant n\left(x_{0}-z\right)
$$

Из (4.4), (4.5) получаем $R(z)-R\left(x_{0}\right)=n\left(x_{0}-z\right)$, а тогда и $\Phi(z)=\Phi\left(x_{0}\right)$, т.е. $z \in X(D)$. Поскольку $\Phi(x)$ - выпуклая функция, то и весь отрезок $\left[x_{0}, z\right] \subset$ $X(D)$. Таким образом, включение (4.3) доказано. Ввиду того, что $X(D) \neq \varnothing$, соотношение (4.2) является следствием вьшеприведенных рассуждений.

Замечание 4.3. Обозначим через $\operatorname{Pr}_{B} A=\{b \in B: \exists a \in A: n(a-b)=$ $\left.\min _{y \in B} n(a-y)\right\}$ проекцию множества $A$ на множество $B$. Легко показать, что утверждение теоремы 4.2 эквивалентно справедливости соотношения

$$
\operatorname{co}\left\{X(D), \operatorname{Pr}_{D} X(D)\right\}=X(D)
$$

Соотношение (4.2) означает, что центр хотя бы одного шара наилучшего приближения содержится в $D$. Сделаем некоторые выводы для случая, когда центр шара наилучшего приближения принадлежит границе множества $D$.

СлЕДСТВИЕ 4.3. Eсли $x_{0} \in X(D) \cap \partial D, m o$

$$
R\left(x_{0}\right)=\min _{x \in D} R(x) .
$$


ДоКАЗАТЕЛЬСТво. Поскольку $x_{0} \in X(D)$, то в соответствии с теоремами 1.1 и 4.1 выполняется включение (4.1). Так как для $x_{0} \in \partial D$ множество $Q^{\rho}\left(x_{0}, \Omega\right)=$ $\left\{x_{0}\right\}$, то, используя формулу (2.23), включение (4.1) можно записать в виде

$$
0_{p} \in \underline{\partial R}\left(x_{0}\right)+\operatorname{co}\left\{v \in-K^{+}\left(x_{0}, D\right): n^{*}(v)=1\right\} .
$$

Отсюда непосредственно следует

$$
\underline{\partial R}\left(x_{0}\right) \cap K^{+}\left(x_{0}, D\right) \neq \varnothing .
$$

Это соотношение, как известно из выпуклого анализа [16; теорема 2.1], эквивалентно (4.6).

СлЕДСТвИЕ 4.4. Пусть int $D=\varnothing u x_{0} \in D$. Для того чтобы точка $x_{0} \in X(D)$, необходимо и достаточно, чтобъ выполнялось (4.6).

ДокАЗАТЕЛЬСтво. Необходимость вытекает из следствия 4.3. Пусть выполняется соотношение (4.6). Поскольку int $D=\varnothing$, то $P(x)=0$ для всех $x \in D$. Поэтому, применяя теорему 4.2, имеем

$$
\Phi\left(x_{0}\right)=R\left(x_{0}\right)=\min _{x \in D} R(x)=\min _{x \in D} \Phi(x)=\min _{x \in \mathbb{R}^{p}} \Phi(x) .
$$

Это означает, что $x_{0} \in X(D)$.

Обозначим через $S n(x, r)=\left\{y \in \mathbb{R}^{p}: n(x-y)=r\right\}$ сферу в норме $n(\cdot)$ с центром в точке $x$ и радиусом $r$.

Лемма 4.1. Если $n(\cdot)$ - строго квазивыпуклая норма, то сферы $\operatorname{Sn}\left(x_{1}, r_{1}\right)$ u $\operatorname{Sn}\left(x_{2}, r_{1}+n\left(x_{1}-x_{2}\right)\right)$ имеют единственную общую точку

$$
y_{0}=x_{1}+r_{1}\left(n\left(x_{1}-x_{2}\right)\right)^{-1}\left(x_{1}-x_{2}\right) .
$$

ДокАЗАТЕльСтво. Если $y \in S n\left(x_{1}, r_{1}\right) \cap S n\left(x_{2}, r_{1}+n\left(x_{1}-x_{2}\right)\right)$, то

$$
n\left(x_{1}-y\right)=r_{1}, \quad n\left(x_{2}-y\right)=r_{1}+n\left(x_{1}-x_{2}\right),
$$

или $n\left(x_{2}-y\right)=n\left(x_{1}-y\right)+n\left(x_{1}-x_{2}\right)$. Это по лемме 2.2 означает, что $x_{2}-y=\lambda\left(x_{1}-y\right)$, где $\lambda>1$. Выражая отсюда $y$ и подставляя в $(4.7)$, получаем единственность общей точки и ее явньй вид.

ТЕОРема 4.3. Если выполняется хотя бъ одно из условий:

1) $n(\cdot)-$ гладкая норма,

2) $n(\cdot)$ - строго квазивыпуклая норма,

3) $p=2$,

то выполняется включение

$$
X(D) \subset D
$$


ДокАЗАтЕльство. Предположим противное, т.е. существует хотя бы одна точка $x_{0} \in X(D)$ такая, что $x_{0} \notin D$. Тогда в соответствии с (4.1) имеет место включение

$$
0_{p} \in \partial \Phi\left(x_{0}\right)=\underline{\partial R}\left(x_{0}\right)+\underline{\partial \rho}_{D}\left(x_{0}\right) .
$$

а) Рассмотрим случай, когда $n(\cdot)$ - гладкая норма. По теореме 2.4 имеем

$$
\underline{\partial \rho}_{D}\left(x_{0}\right)=n^{\prime}\left(x_{0}-z\right) \quad \forall z \in Q^{\rho}\left(x_{0}, D\right) \text {. }
$$

Покажем, что сушествует элемент $z^{*} \in Q^{R}\left(x_{0}, D\right)$ такой, что

$$
n^{\prime}\left(x_{0}-z\right)=-n^{\prime}\left(x_{0}-z^{*}\right) \quad \forall z \in Q^{\rho}\left(x_{0}, D\right) \text {. }
$$

Включение (4.9), как следует из (2.5) и (4.10), означает, что существуют натуральное $m$, элементы $\left\{z_{i}\right\}_{i=1, \ldots, m} \subset Q^{R}\left(x_{0}, D\right)$, числа $\alpha_{i}>0, i=1, \ldots, m$, такие, что $\sum_{i=1}^{m} \alpha_{i}=1$ и

$$
n^{\prime}\left(x_{0}-z\right)=-\sum_{i=1}^{m} \alpha_{i} n^{\prime}\left(x_{0}-z_{i}\right), \quad z \in Q^{\rho}\left(x_{0}, D\right) .
$$

Поскольку $n^{\prime}\left(x_{0}-z_{i}\right)=\partial n\left(x_{0}-z_{i}\right)$, то из (2.1) следует

$$
n^{*}\left(n^{\prime}\left(x_{0}-z_{i}\right)\right)=1 \text {. }
$$

Поэтому, учитывая также то, что

$$
n(x)=\max _{n^{*}(v) \leqslant 1}\langle v, x\rangle,
$$

имеем

$$
\left\langle n^{\prime}\left(x_{0}-z_{i}\right), x_{0}-z\right\rangle \leqslant n\left(x_{0}-z\right) .
$$

Кроме того, в соответствии с (2.1) выполняется

$$
\left\langle n^{\prime}\left(x_{0}-z\right), x_{0}-z\right\rangle=n\left(x_{0}-z\right) .
$$

Тогда из (4.12), (4.15), (4.16) следует, что

$$
\left\langle-n^{\prime}\left(x_{0}-z_{i}\right), x_{0}-z\right\rangle=n\left(x_{0}-z\right), \quad i=1, \ldots, m, \quad z \in Q^{\rho}\left(x_{0}, D\right) .
$$

Сравнивая (2.1) с (4.13) и (4.17), приходим к выводу

$$
-n^{\prime}\left(x_{0}-z_{i}\right) \subset \partial n\left(x_{0}-z\right)=n^{\prime}\left(x_{0}-z\right),
$$

т.е. $-n^{\prime}\left(x_{0}-z_{i}\right)=n^{\prime}\left(x_{0}-z\right), i=1, \ldots, m, z \in Q^{\rho}\left(x_{0}, D\right)$. Тем самым (4.11) доказано.

Зафиксируем точку $z_{0} \in Q^{\rho}\left(x_{0}, D\right)$. Шар $B n\left(x_{0}, \rho_{D}\left(x_{0}\right)\right)$ касается выпуклого множества $D$ в точке $z_{0}$. Поэтому через нее проходит гиперплоскость, разделяющая множества $B n\left(x_{0}, \rho_{D}\left(x_{0}\right)\right)$ и $D$. Поскольку в рассматриваемом случае шар 
имеет гладкую границу и $n^{\prime}\left(x_{0}-z_{0}\right)$ является нормалью к нему в точке $z_{0}$, то эта гиперплоскость имеет вид

$$
\pi=\left\{x \in R^{p}:\left\langle x, n^{\prime}\left(x_{0}-z_{0}\right)\right\rangle=\left\langle z_{0}, n^{\prime}\left(x_{0}-z_{0}\right)\right\rangle\right\} .
$$

При этом вьполняется неравенство

$$
\left\langle x, n^{\prime}\left(x_{0}-z_{0}\right)\right\rangle \leqslant\left\langle y, n^{\prime}\left(x_{0}-z_{0}\right)\right\rangle \quad \forall x \in D, \quad y \in B n\left(x_{0}, \rho_{D}\left(x_{0}\right)\right) .
$$

Так как $Q^{R}\left(x_{0}, D\right) \subset D, x_{0} \in B n\left(x_{0}, \rho_{D}\left(x_{0}\right)\right)$, то из (4.18) получаем

$$
\left\langle z-x_{0}, n^{\prime}\left(x_{0}-z_{0}\right)\right\rangle \leqslant 0 \quad \forall z \in Q^{R}\left(x_{0}, D\right) .
$$

Теперь, подставляя сюда точку $z^{*} \in Q^{R}\left(x_{0}, D\right)$, удовлетворяюшую $(4.11)$, и используя (2.1), приходим к противоречию

$$
\left\langle z^{*}-x_{0},-n^{\prime}\left(x_{0}-z^{*}\right)\right\rangle=n\left(x_{0}-z^{*}\right) \leqslant 0 .
$$

б) Пусть $n(\cdot)$ - строго квазивыпуклая норма. Возьмем любую точку $z_{0} \in$ $Q^{\rho}\left(x_{0}, D\right)$. По теореме 4.2 имеем $\left[x_{0}, z_{0}\right] \subset X(D)$ и, следовательно, $\Phi\left(x_{0}\right)=\Phi\left(z_{0}\right)$. А так как $\Phi\left(x_{0}\right)=R\left(x_{0}\right)+\rho_{D}\left(x_{0}\right)=R\left(x_{0}\right)+n\left(x_{0}-z_{0}\right)$ и $\Phi\left(z_{0}\right)=R\left(z_{0}\right)$, то получаем $R\left(z_{0}\right)=R\left(x_{0}\right)+n\left(x_{0}-z_{0}\right)$. Это означает, что $B n\left(x_{0}, R\left(x_{0}\right)\right) \subset B n\left(z_{0}, R\left(z_{0}\right)\right)$, причем граничные сферы этих шаров $S n\left(x_{0}, R\left(x_{0}\right)\right)$ и $S n\left(z_{0}, R\left(z_{0}\right)\right)$ по лемме 4.1 касаются в единственной точке $y_{0}=x_{0}+R\left(x_{0}\right)\left(n\left(x_{0}-z_{0}\right)\right)^{-1}\left(x_{0}-z_{0}\right)$. Из определения функции $R(\cdot)$ следует, что множество $D$ имеет общие точки с $\operatorname{Sn}\left(x_{0}, R\left(x_{0}\right)\right)$ и $\operatorname{Sn}\left(z_{0}, R\left(z_{0}\right)\right)$. Поэтому приходим к выводу, что $y_{0} \in D$.

Выпуклые компакты $D$ и $B n\left(x_{0}, \rho_{D}\left(x_{0}\right)\right)$ не имеют общих внутренних точек. Следовательно, по теореме о разделяющей гиперплоскости найдется элемент $g_{0} \in \mathbb{R}^{p}, g_{0} \neq 0_{p}$, такой, что (см., например, [12; теорема 1.3])

$$
\left\langle x, g_{0}\right\rangle \leqslant\left\langle z_{0}, g_{0}\right\rangle \leqslant\left\langle y, g_{0}\right\rangle \quad \forall x \in B n\left(x_{0}, \rho_{D}\left(x_{0}\right)\right), \quad y \in D .
$$

Так как $x_{0} \in \operatorname{int} B n\left(x_{0}, \rho_{D}\left(x_{0}\right)\right)$, то $\left\langle x_{0}, g_{0}\right\rangle<\left\langle z_{0}, g_{0}\right\rangle$. Поэтому, учитывая $y_{0} \in D$, получаем

$$
\left\langle y_{0}, g_{0}\right\rangle=\left\langle x_{0}, g_{0}\right\rangle+\frac{R\left(x_{0}\right)}{n\left(x_{0}-z_{0}\right)}\left\langle x_{0}-z_{0}, g_{0}\right\rangle<\left\langle z_{0}, g_{0}\right\rangle .
$$

Это противоречит правой части неравенства (4.19).

в) Пусть размерность пространства $p=2$. Из (4.9) следует сушествование элемента $v_{0} \in \underline{\partial R}\left(x_{0}\right) \cap-\underline{\partial \rho}{ }_{D}\left(x_{0}\right)$.

Включение $v_{0} \in-\underline{\partial \rho}{ }_{D} \overline{\left(x_{0}\right)}$ в соответствии с формулами (2.15) и (2.1) означает вьполнение для любого $z_{0} \in Q^{\rho}\left(x_{0}, D\right)$ соотношений

$$
\begin{gathered}
n^{*}\left(v_{0}\right)=1, \\
v_{0} \in K^{+}\left(z_{0}, D\right), \\
\left\langle v_{0}, z_{0}-x_{0}\right\rangle=n\left(x_{0}-z_{0}\right) .
\end{gathered}
$$


Теперь покажем, что при $p=2$ из включения $v_{0} \in \underline{\partial R}\left(x_{0}\right)$ и (4.20) следует существование элементов $z_{1}, z_{2} \in Q^{R}\left(x_{0}, D\right)$ и соответствующих им $v_{1} \in \partial n\left(x_{0}-z_{1}\right)$, $v_{2} \in \partial n\left(x_{0}-z_{2}\right)$ и $\alpha \in[0,1]$ таких, что

$$
v_{0}=\alpha v_{1}+(1-\alpha) v_{2}
$$

Действительно, из (2.5) по теореме Каратеодори (см., например, [12; теорема 1.1]) следует сушествование элементов $\left\{z_{i}\right\}_{i=1,2,3} \subset Q^{R}\left(x_{0}, D\right)$, не обязательно разных, и соответствующих элементов $v_{i} \in \partial n\left(x_{0}-z_{i}\right), i=1,2,3$, отличных друг от друга, и чисел $\alpha_{i} \geqslant 0, i=1,2,3, \sum_{i=1}^{3} \alpha_{i}=1$, таких, что $v_{0}=\alpha_{1} v_{1}+\alpha_{2} v_{2}+\alpha_{3} v_{3}$. При этом в силу $(2.1) n^{*}\left(v_{i}\right)=1, i=1,2,3$. Если бы элементы $\left\{v_{i}\right\}_{i=1,2,3}$ не лежали на одной прямой и все $\alpha_{i}>0$, то это влекло бы $v_{0} \in \operatorname{int} \operatorname{co}\left\{v_{1}, v_{2}, v_{3}\right\}$, а следовательно, и $n^{*}\left(v_{0}\right)<1$, что противоречило бы (4.20). Поэтому без ограничения общности можно считать $\alpha_{3}=0$, что приводит к возможности представления элемента $v_{0}$ в виде $(4.23)$.

Из (4.21) следует, что

$$
\left\langle v_{0}, x-z_{0}\right\rangle \geqslant 0 \quad \forall x \in D
$$

и, значит, $\left\langle v_{0}, z_{i}-x_{0}\right\rangle+\left\langle v_{0}, x_{0}-z_{0}\right\rangle \geqslant 0, i=1,2$. Ввиду (4.22) последнее эквивалентно тому, что

$$
\left\langle v_{0}, z_{i}-x_{0}\right\rangle \geqslant n\left(x_{0}-z_{0}\right), \quad i=1,2 .
$$

Для $v_{i} \in \partial n\left(x_{0}-z_{i}\right)$ в соответствии с (2.1) имеем

$$
\left\langle v_{i}, z_{i}-x_{0}\right\rangle=-n\left(z_{i}-x_{0}\right), \quad n^{*}\left(v_{i}\right)=1, \quad i=1,2 .
$$

Тогда, подставляя (4.23) в (4.24) и используя (4.25), получаем

$$
\begin{array}{r}
-\alpha n\left(z_{1}-x_{0}\right)+(1-\alpha)\left\langle v_{2}, z_{1}-x_{0}\right\rangle \geqslant n\left(x_{0}-z_{0}\right), \\
\alpha\left\langle v_{1}, z_{2}-x_{0}\right\rangle-(1-\alpha) n\left(z_{2}-x_{0}\right) \geqslant n\left(x_{0}-z_{0}\right) .
\end{array}
$$

Но поскольку $n^{*}\left(v_{i}\right)=1, i=1,2$, то в силу (4.14)

$$
\left\langle v_{2}, z_{1}-x_{0}\right\rangle \leqslant n\left(z_{1}-x_{0}\right), \quad\left\langle v_{2}, z_{2}-x_{0}\right\rangle \leqslant n\left(z_{2}-x_{0}\right) .
$$

Отсюда следует, что при $\alpha \in\left[\frac{1}{2}, 1\right]$ неравенство (4.26) становится противоречивьм. При $\alpha \in\left[0, \frac{1}{2}\right]$ противоречивым становится неравенство (4.27). Теорема доказана полностью.

Приведем пример, когда $X(D) \not \subset D$. 
ПримеР 4.1. Пусть $p=3, x=\left(x^{(1)}, x^{(2)}, x^{(3)}\right) \in \mathbb{R}^{3}, n(x)=\max _{i=1,2,3}\left|x^{(i)}\right|$, $D=\operatorname{co}\left\{z_{1}, z_{2}, z_{3},\right\}$, где $z_{1}=(1,1,1), z_{2}=(1,-1,-1), z_{3}=(-1,-1,1)$. Очевидно, $x_{0}=(0,0,0) \notin D$ и $Q^{\rho}\left(x_{0}, D\right)=\left\{z_{0}\right\}$, где $z_{0}=\left(\frac{1}{3},-\frac{1}{3}, \frac{1}{3}\right)$. Используя формулу (2.1), нетрудно получить

$$
\begin{aligned}
& \partial n\left(x_{0}-z_{0}\right)=\operatorname{co}\{(-1,0,0),(0,1,0),(0,0,-1)\}, \\
& \partial n\left(x_{0}-z_{1}\right)=\operatorname{co}\{(-1,0,0),(0,-1,0),(0,0,-1)\}, \\
& \partial n\left(x_{0}-z_{2}\right)=\operatorname{co}\{(-1,0,0),(0,1,0),(0,0,1)\} \\
& \partial n\left(x_{0}-z_{3}\right)=\operatorname{co}\{(1,0,0),(0,1,0),(0,0,-1)\} .
\end{aligned}
$$

Поскольку $\left\{z_{1}, z_{2}, z_{3}\right\} \subset Q^{R}\left(x_{0}, D\right)$, то в силу формулы $(2.5) \operatorname{co}\left\{\partial n\left(x_{0}-z_{i}\right)\right.$ : $i=1,2,3\} \subset \underline{\partial R}\left(x_{0}\right)$. Поэтому, подставляя сюда $(4.28)$, имеем

$$
\operatorname{co}\{(1,0,0),(0,-1,0),(0,0,1)\} \subset \underline{\partial R}\left(x_{0}\right) .
$$

Нетрудно подсчитать, что $K^{+}\left(z_{0}, D\right)=\left\{x=(\lambda,-\lambda, \lambda) \in \mathbb{R}^{3}: \lambda \in \mathbb{R}^{1}\right\}$. Тогда $-\partial \rho_{D}\left(x_{0}\right)=\partial n\left(z_{0}-x_{0}\right) \cap K^{+}\left(z_{0}, D\right)=\left\{y_{0}\right\}$, где $y_{0}=\left(\frac{1}{3},-\frac{1}{3}, \frac{1}{3}\right)$. Так как эту точку можно представить в виде

$$
y_{0}=\frac{1}{3}(1,0,0)+\frac{1}{3}(0,-1,0)+\frac{1}{3}(0,0,1),
$$

то, учитывая (4.29), мы приходим к выводу, что $y_{0} \in \underline{\partial R}\left(x_{0}\right) \cap-\underline{\partial \rho}{ }_{D}\left(x_{0}\right)$ или $0_{3} \in \underline{\partial R}\left(x_{0}\right)+\underline{\partial \rho}{ }_{D}\left(x_{0}\right)$. Следовательно, в соответствии с (4.1) точка $x_{0} \in X(D)$, а также по теореме 4.2 и весь отрезок $\left[x_{0}, z_{0}\right] \subset X(D)$. Но так как $x_{0} \notin D$, то $X(D) \not \subset D$.

Рассмотренный пример одновременно показывает, что решение задачи (1.1) может быть не единственным. Приведем условие, при котором решение является единственным.

Нетрудно увидеть справедливость следующего факта.

Лемма 4.2. Если $x_{1}, x_{2} \in X(D)$, то выпуклье функиии $R(x)$ и $P(x)$ являются аффинными на $\left[x_{1}, x_{2}\right]$.

ТЕОРема 4.4. Если $n(\cdot)$ - строго квазивыпуклая норма, то задача (1.1) имеет единственное решение.

ДокАЗАТЕльство. Предположим, что решение не единственно и существует по крайней мере два шара наилучшего приближения с центрами $x_{1}, x_{2} \in X(D)$. По теореме 4.3 выполняется включение $X(D) \subset D$ и так как $\left[x_{1}, x_{2}\right] \subset X(D)$, то

$$
\Phi(x)=R(x)-\rho_{\Omega}(x)=\min _{x \in \mathbb{R}^{p}} \Phi(x)=\Phi\left(x_{1}\right) \quad \forall x \in\left[x_{1}, x_{2}\right] .
$$

Будем для определенности считать, что $R\left(x_{2}\right) \geqslant R\left(x_{1}\right)$. Так как функция $R(x)$ ведет себя на отрезке $\left[x_{1}, x_{2}\right]$ по лемме 4.2 как аффинная функция, то в силу теоремы 2.3

$$
R\left(x_{2}\right)=R\left(x_{1}\right)+n\left(x_{1}-x_{2}\right) .
$$


Из (4.30), (4.31) получаем, что и

$$
\rho_{\Omega}\left(x_{2}\right)=\rho_{\Omega}\left(x_{1}\right)+n\left(x_{1}-x_{2}\right) \text {. }
$$

Теперь из (4.32) следует

$$
R\left(x_{1}\right)>\rho_{\Omega}\left(x_{1}\right) \text {. }
$$

Действительно, предположим, что $R\left(x_{1}\right)=\rho_{\Omega}\left(x_{1}\right)$. Тогда из включений

$$
B n\left(x_{1}, \rho_{\Omega}\left(x_{1}\right)\right) \subset D \subset B n\left(x_{1}, R\left(x_{1}\right)\right)
$$

получаем $D=B n\left(x_{1}, \rho_{\Omega}\left(x_{1}\right)\right)$ и, следовательно, $\rho_{\Omega}\left(x_{1}\right)=\max _{x \in D} \rho_{\Omega}(x)$. Это противоречит (4.32).

Из (4.31) и леммы 4.1 получаем, что граничные сферы вложенных шаров $B n\left(x_{1}, R\left(x_{1}\right)\right)$ и $B n\left(x_{2}, R\left(x_{2}\right)\right)$ касаются в единственной точке

$$
y_{0}=x_{1}+\frac{x_{1}-x_{2}}{n\left(x_{1}-x_{2}\right)} R\left(x_{1}\right) .
$$

При этом поскольку выполняются включения

$$
D \subset B n\left(x_{1}, R\left(x_{1}\right)\right) \subset B n\left(x_{2}, R\left(x_{2}\right)\right)
$$

и шар $B n\left(x_{2}, R\left(x_{2}\right)\right)$ должен иметь общую точку с $D$, то $y_{0} \in D$. А так как в силу (4.32) точка $x_{2} \in \operatorname{int} D$, то

$$
\alpha x_{2}+(1-\alpha) y_{0} \in \operatorname{int} D \quad \forall \alpha \in(0,1)
$$

С другой стороны, из (4.32) и леммы 4.1 получаем, что граничные сферы вложенных шаров $B n\left(x_{1}, \rho_{\Omega}\left(x_{1}\right)\right)$ и $B n\left(x_{2}, \rho_{\Omega}\left(x_{2}\right)\right)$ касаются в единственной точке

$$
y_{1}=x_{1}+\frac{x_{1}-x_{2}}{n\left(x_{1}-x_{2}\right)} \rho_{\Omega}\left(x_{1}\right) \text {. }
$$

При этом поскольку выполняются включения

$$
B n\left(x_{1}, \rho_{\Omega}\left(x_{1}\right)\right) \subset B n\left(x_{2}, \rho_{\Omega}\left(x_{2}\right)\right) \subset D
$$

и шар $B n\left(x_{1}, \rho_{\Omega}\left(x_{1}\right)\right)$ имеет обшую точку с множеством $\Omega$, то

$$
y_{1} \in \Omega
$$

Из $(4.33),(4.34)$ и $(4.36)$ следует, что сушествует $\alpha_{0} \in(0,1)$ такое, что $y_{1}=\alpha_{0} x_{2}+$ $\left(1-\alpha_{0}\right) y_{0}$. Это с учетом (4.35) противоречит (4.37). 
ЗАмечАниЕ 4.4. Строгая квазивыпуклость нормы означает строгую выпуклость ее шаров. Как мы доказали, наилучшее приближение вьпуклого компакта шаром такой нормы имеет единственное решение. А будет ли всегда единственньм решение задачи о наилучшем приближении строго выпуклого компакта шаром нормы, которая не является строго квазивыпуклой? Следующий пример дает отрицательный ответ на этот вопрос.

ПримеР 4.2. Пусть $p=2, x=\left(x^{(1)}, x^{(2)}\right) \in \mathbb{R}^{2}, n(x)=\max \left\{\left|x^{(1)}\right| ;\left|x^{(2)}\right|\right\}$, $D=\left\{x \in \mathbb{R}^{2}:\left(x^{(1)}+1\right)^{2}+\left(x^{(2)}-1\right)^{2} \leqslant 4,\left(x^{(1)}-2\right)^{2}+\left(x^{(2)}+2\right)^{2} \leqslant 10\right\}$ - строго вьпуклый компакт, $x_{0}=(0,0), x_{1}=((1+\sqrt{2}-\sqrt{5}) / 2,(\sqrt{5}-\sqrt{2}-1) / 2)$.

а) Нетрудно проверить, что $Q^{R}\left(x_{0}, D\right)=\left\{z_{1} ; z_{2}\right\}$, где $z_{1}=(-1,-1), z_{2}=(1,1)$, и $Q^{\rho}\left(x_{0}, \Omega\right)=\left\{z_{0}\right\}$, где $z_{0}=(2-\sqrt{5}, \sqrt{5}-2)$. Учитывая, что $n^{*}(x)=\left|x^{(1)}\right|+\left|x^{(2)}\right|$, и используя $(2.1)$, имеем

$$
\begin{gathered}
\partial n\left(x_{0}-z_{0}\right)=\operatorname{co}\{(1,0),(0,-1)\}, \quad \partial n\left(x_{0}-z_{1}\right)=\operatorname{co}\{(1,0),(0,1)\} \\
\partial n\left(x_{0}-z_{2}\right)=\operatorname{co}\{(-1,0),(0,-1)\} \\
K^{+}\left(z_{0}, D\right)=\left\{x \in \mathbb{R}^{2}: x^{(1)}=-x^{(2)}, x^{(1)} \geqslant 0\right\} .
\end{gathered}
$$

Используя формулы (2.5) и (2.16), получаем

$$
\begin{gathered}
\underline{\partial R}\left(x_{0}\right)=\operatorname{co}\left\{\partial n\left(x_{0}-z_{1}\right), \partial n\left(x_{0}-z_{2}\right)\right\}=\left\{x \in \mathbb{R}^{2}:\left|x^{(1)}\right|+\left|x^{(2)}\right| \leqslant 1\right\}, \\
\overline{\partial \rho_{\Omega}}\left(x_{0}\right)=\left\{\left(\frac{1}{2},-\frac{1}{2}\right)\right\} .
\end{gathered}
$$

Так как $x_{0} \in \operatorname{int} D$, то $\underline{\partial P}\left(x_{0}\right)=-\overline{\partial \rho}_{\Omega}\left(x_{0}\right)$. Поэтому из (4.38) имеем $0_{2} \in \underline{\partial R}\left(x_{0}\right)+$ $\underline{\partial P}\left(x_{0}\right) \equiv \underline{\partial \Phi}\left(x_{0}\right)$, что означает, что $x_{0} \in X(D)$, т.е. $x_{0}$ - центр шара наилучшего приближения.

б) Также проверяем, что $Q^{R}\left(x_{1}, D\right)=\left\{z_{1} ; z_{2}\right\}$ и $Q^{\rho}\left(x_{1}, \Omega\right)=\left\{z_{0} ; z_{3}\right\}$, где $z_{3}=(\sqrt{2}-1,1-\sqrt{2})$. При этом

$$
\begin{array}{rlrl}
\partial n\left(x_{1}-z_{0}\right) & =\operatorname{co}\{(1,0),(0,-1)\}, & & \partial n\left(x_{1}-z_{3}\right)=\operatorname{co}\{(-1,0),(0,-1)\}, \\
\partial n\left(x_{1}-z_{1}\right) & =\{(1,0)\}, & & \partial n\left(x_{1}-z_{2}\right)=\{(0,-1)\}, \\
& K^{+}\left(z_{3}, D\right)=\left\{x \in \mathbb{R}^{2}: x^{(1)}=-x^{(2)}, x^{(1)} \leqslant 0\right\} .
\end{array}
$$

Теперь, используя (2.5) и (2.16), получаем

$$
\begin{aligned}
\underline{\partial R}\left(x_{1}\right) & =\operatorname{co}\left\{\partial n\left(x_{1}-z_{1}\right), \partial n\left(x_{1}-z_{2}\right)\right\}=\operatorname{co}\{(1,0) ;(0,-1)\} \\
\overline{\partial \rho_{\Omega}}\left(x_{1}\right) & =\operatorname{co}\left\{v \in K^{+}\left(z_{0}, D\right) \cup K^{+}\left(z_{3}, D\right): n^{*}(v)=1\right\} \\
& =\operatorname{co}\left\{\left(-\frac{1}{2}, \frac{1}{2}\right) ;\left(\frac{1}{2},-\frac{1}{2}\right)\right\}
\end{aligned}
$$

Далее, как и в п. а), приходим к выводу, что $0_{2} \in \underline{\partial \Phi}\left(x_{1}\right)$, т.е. точка $x_{1}$ также является центром шара наилучшего приближения.

Таким образом, решение задачи в данном примере не единственно. 
ЗАмЕчАнИЕ 4.5. Примеры показывают, что гладкость нормы не гарантирует единственность решения задачи.

ЗАмЕчАниЕ 4.6. Для случая евклидовой нормы в [2] доказано, что решение задачи (1.1) единственно и центр шара наилучшего приближения содержится в $D$, т.е. $X(D)=\left\{x_{0}\right\}, x_{0} \in D$. Поскольку евклидова норма является строго квазивьпуклой, то эти факты мы можем истолковать как следствия из теорем 4.3 и 4.4. В [2], кроме того, доказано, что если int $D \neq \varnothing$, то $x_{0} \in$ int $D$. Этот факт нетрудно также получить непосредственно из условия оптимальности (4.1). В целом, применяя теорему 4.1 и учитывая, что для евклидовой нормы $n^{\prime}(x)=x\|x\|^{-1}$ при $x \neq 0_{p}$, получаем

СлЕДСТВИЕ 4.5. Пусть int $D \neq \varnothing, a n(\cdot)=\|\cdot\|-$ евклидова норма. Для того чтобы точка $x_{0}$ была иентром шара наилучшего приближсения, необходимо и достаточно, чтобы $x_{0} \in \operatorname{int} D$ и выполнялось соотношение

$$
0_{p} \in \operatorname{co}\left\{\frac{x_{0}-y}{\left\|x_{0}-y\right\|}: y \in Q^{R}\left(x_{0}, D\right)\right\}-\operatorname{co}\left\{\frac{x_{0}-z}{\left\|x_{0}-z\right\|}: z \in Q^{\rho}\left(x_{0}, \Omega\right)\right\}
$$

ЗАмечАниЕ 4.7. Если int $D \neq \varnothing$, то необходимое условие решения задачи (1.1), полученное в [2; теорема 19] для евклидовой нормы, в наших обозначениях принимает вид

$$
0_{p} \in \operatorname{co}\left(\left\{y-x_{0}: y \in Q^{R}\left(x_{0}, D\right)\right\} \cup\left\{x_{0}-z: z \in Q^{\rho}\left(x_{0}, \Omega\right)\right\}\right)
$$

Легко видеть, что соотношение (4.40) вытекает из (4.39). Однако эти соотношения не эквивалентны. Действительно, предположим, что точка $x_{0}$ является центром наименьшего шара, содержащего $D$, или центром наибольшего шара, содержашегося в $D$. Поскольку в силу $(2.5)$ и (2.16)

$$
\begin{gathered}
\underline{\partial R}(x)=\operatorname{co}\left\{\frac{x-y}{\|x-y\|}: y \in Q^{R}(x, D)\right\}, \\
\overline{\partial \rho}_{\Omega}(x)=\operatorname{co}\left\{\frac{x-z}{\|x-z\|}: z \in Q^{\rho}\left(x_{0}, \Omega\right)\right\},
\end{gathered}
$$

то точка $x_{0}$ удовлетворяет в этих случаях соответственно включениям

$$
0_{p} \in \operatorname{co}\left\{x_{0}-y: y \in Q^{R}\left(x_{0}, D\right)\right\}, \quad 0_{p} \in \operatorname{co}\left\{x_{0}-z: z \in Q^{\rho}\left(x_{0}, \Omega\right)\right\}
$$

Следовательно, точка $x_{0}$ будет в этих случаях удовлетворять соотношению (4.40). Однако легко привести пример, когда такая точка не является центром шара наилучшего приближения. Это говорит о том, что соотношение (4.40) достаточным условием решения задачи (1.1) не является. 
ЗАмечаниЕ 4.8. Пусть $p=2, D$ - треугольник, а $n(\cdot)$ - евклидова норма. Пользуясь соотношением (4.39), нетрудно доказать, что точка $x_{0}$, являюшаяся центром шара (круга) наилучшего приближения, есть пересечение перпендикуляра к наибольшей стороне треугольника, проведенного в ее середине, и биссектрисы прилежашего к этой стороне наименьшего угла. Радиус искомого круга есть $r_{0}=\left(R\left(x_{0}\right)+\rho_{\Omega}\left(x_{0}\right)\right) / 2$.

Обозначим через $K v\left(\mathbb{R}^{p}\right)$ пространство всех непустых вьпуклых компактов из $\mathbb{R}^{p}$. Нетрудно убедиться, что расстояние Хаусдорфа $h(A, B)$, индуцированное любой нормой $n(\cdot)$, является метрикой в $K v\left(\mathbb{R}^{p}\right)$. Обобщая соответствующие результаты из [2], рассмотрим некоторые свойства функции

$$
h_{0}(D)=\min _{x \in \mathbb{R}^{p}, r \geqslant 0} h(D, B n(x, r)): K v\left(\mathbb{R}^{p}\right) \rightarrow \mathbb{R}^{1}
$$

и многозначного отображения $X(D): K v\left(\mathbb{R}^{p}\right) \rightarrow 2^{\mathbb{R}^{p}}$.

ТЕОРема 4.5. Функция $h_{0}(D)$ является липиицевой на $K v\left(\mathbb{R}^{p}\right)$ с константой, равной единиче, т.е.

$$
\left|h_{0}\left(D_{1}\right)-h_{0}\left(D_{2}\right)\right| \leqslant h\left(D_{1}, D_{2}\right) \quad \forall D_{1}, D_{2} \in K v\left(\mathbb{R}^{p}\right) .
$$

ДокАЗАТЕЛЬСтво. Пусть шар $B n\left(x_{1}, r_{1}\right)$ является шаром наилучшего приближения для $D_{1}$, а шар $B n\left(x_{2}, r_{2}\right)$ для $D_{2}$. Тогда, используя свойства метрики, имеем

$$
\begin{aligned}
h_{0}\left(D_{1}\right) & =h\left(D_{1}, B n\left(x_{1}, r_{1}\right)\right) \leqslant h\left(D_{1}, B n\left(x_{2}, r_{2}\right)\right) \\
& \leqslant h\left(D_{1}, D_{2}\right)+h\left(D_{2}, B n\left(x_{2}, r_{2}\right)\right)=h\left(D_{1}, D_{2}\right)+h_{0}\left(D_{2}\right) .
\end{aligned}
$$

То есть получаем неравенство

$$
h_{0}\left(D_{1}\right)-h_{0}\left(D_{2}\right) \leqslant h\left(D_{1}, D_{2}\right)
$$

Аналогично получаем и неравенство

$$
h_{0}\left(D_{2}\right)-h_{0}\left(D_{1}\right) \leqslant h\left(D_{1}, D_{2}\right) .
$$

Из (4.42), (4.43) следует (4.41).

Tеорема 4.6. Многозначное отображение $X(D): K v\left(\mathbb{R}^{p}\right) \rightarrow 2^{\mathbb{R}^{p}}$ полунепрерывно сверху.

ДоказАтельство. Зафиксируем $D_{0} \in K v\left(\mathbb{R}^{p}\right)$ и обозначим через $K$ множество элементов $D \in K v\left(\mathbb{R}^{p}\right)$, удовлетворяющих неравенству $h\left(D, D_{0}\right) \leqslant 1$. Нетрудно видеть, что сушествует конечное число $\lambda$ такое, что

$$
\|x\|+r<\lambda \quad \forall x \in X(D), \quad r=(R(x)-P(x)) / 2, \quad D \in K .
$$

Возьмем произвольную последовательность $\left\{D_{i}\right\} \subset K v\left(\mathbb{R}^{p}\right), i=1,2, \ldots$, такую, что $h\left(D_{i}, D_{0}\right) \rightarrow 0, i \rightarrow \infty$. В силу (4.44) можно выбрать последовательности 
$x_{i} \in X\left(D_{i}\right), r_{i}=\left(R\left(x_{i}\right)-P\left(x_{i}\right)\right) / 2$ такие, что $x_{i} \rightarrow x^{*}, r_{i} \rightarrow r^{*}, i \rightarrow \infty$. Покажем, что $x^{*} \in X\left(D_{0}\right)$.

Предположим противное, т.е.

$$
h\left(D_{0}, B n\left(x^{*}, r^{*}\right)\right)-h_{0}\left(D_{0}\right)=\delta>0 .
$$

Отсюда следует, что при достаточно больших $i$

$$
h\left(D_{i}, B n\left(x_{i}, r_{i}\right)\right)-h\left(D_{i}, B n\left(x_{0}, r_{0}\right)\right) \geqslant \frac{\delta}{2}>0
$$

где $x_{0} \in X\left(D_{0}\right), r_{0}=\left(R\left(x_{0}\right)-P\left(x_{0}\right)\right) / 2$, т.е. $B n\left(x_{0}, r_{0}\right)$ - один из шаров наилучшего приближения для $D_{0}$. Но (4.45) противоречит тому, что $B n\left(x_{i}, r_{i}\right)$-шар наилучшего приближения для $D_{i}$.

СЛЕДСТВИЕ 4.6. Если $n(\cdot)$ - строго квазивыпуклая норма, то отображсение $X(D)$ является однозначнылм и непрерывным на $K v\left(\mathbb{R}^{p}\right)$.

ЗАмЕчАниЕ 4.9. Нижеследующий пример показывает, что м.о. $X(D): K v\left(\mathbb{R}^{p}\right)$ $\rightarrow 2^{\mathbb{R}^{p}}$ может не являться полунепрерывным снизу.

ПрИМЕР 4.3. Пусть $p=3, x=\left(x^{(1)}, x^{(2)}, x^{(3)}\right) \in \mathbb{R}^{3}$,

$$
\begin{gathered}
n(x)=\max \left\{\left|x^{(1)}\right| ;\left|x^{(2)}\right| ;\left|x^{(3)}\right|\right\} \\
D_{0}=\operatorname{co}\left\{\left(0,1, \frac{1}{2}\right),\left(0,1,-\frac{1}{2}\right),\left(0,-1, \frac{1}{2}\right),\left(0,-1,-\frac{1}{2}\right)\right\} .
\end{gathered}
$$

Поскольку int $D_{0}=\varnothing$ и компакт $D_{0}$ обладает центральной симметрией относительно точки $x_{0}=(0,0,0)$, то в соответствии со следствием 4.1 шар $B n\left(0_{3}, \frac{1}{2}\right)$ является шаром наилучшего приближения для $D_{0}$ и

$$
h_{0}\left(D_{0}\right)=h\left(D_{0}, B n\left(0_{3}, \frac{1}{2}\right)\right)=\frac{1}{2}
$$

Однако легко увидеть, что для $x \in \operatorname{co}\left\{\left(0,0, \frac{1}{2}\right),\left(0,0,-\frac{1}{2}\right)\right\}$ выполняется

$$
h\left(D_{0}, B n\left(x, \frac{1}{2}\right)\right)=\frac{1}{2}
$$

т.е. шары $B n\left(x, \frac{1}{2}\right)$ также являются шарами наилучшего приближения. Следовательно,

$$
\operatorname{co}\left\{\left(0,0, \frac{1}{2}\right),\left(0,0,-\frac{1}{2}\right)\right\} \subset X\left(D_{0}\right) .
$$

Возьмем в качестве $D_{i}=\operatorname{co}\left\{D_{0},\left(\frac{1}{i}, 0,0\right),\left(-\frac{1}{i}, 0,0\right)\right\}$. Очевидно, что $h\left(D_{0}, D_{i}\right)$ $\rightarrow 0, i \rightarrow \infty$, и компакты $D_{i}$ обладают центральной симметрией относительно 
той же точки $x_{0}=(0,0,0)$. Следовательно, $x_{0} \in X\left(D_{i}\right)$. Но при этом $x_{0}$ является центром шара наименьшего радиуса, содержащего множество $D_{i}$ и, главное, $x_{0}$ - единственная точка максимума функции $\rho_{\Omega_{i}}(x)$ на $\Omega_{i}=\overline{\mathbb{R}^{3} \backslash D_{i}}$. Поэтому $X\left(D_{i}\right)=\left\{x_{0}\right\}$. Это означает, что элементы из $X\left(D_{0}\right)$, отличные от точки $x_{0}$, не могут быть предельными точками элементов из $X\left(D_{i}\right)$. Таким образом, в данном случае полунепрерьвности снизу м.о. $X(D)$ нет.

Авторы выражают глубокую признательность М. С. Никольскому за полезное обсуждение результатов работы.

\section{Список литературы}

1. Черноусько Ф. Л. Оценивание фазового состояния динамических систем: метод эллипсоидов. М.: Наука, 1988.

2. Никольский М. С., Силин Д. Б. О наилучшем приближении вьпуклого компакта элементами аддиала // Труды МИРАН. 1995. Т. 211. С. 338-354.

3. D'Ocagne M. Sur certaine figures minimales // Bull. Soc. Math. France. 1884. V. 12. P. $168-177$.

4. Lebesgue H. Sur quelques questions de minimum, relatives and courbes orbiformes, et sur leurs rapports avec le calcul des variations // J. Math. Pures Appl. 1921. V. 4. P. 67-96.

5. Bonnesen T., Fenchel W. Theory der konvexen Korper. Berlin: Springer-Verlag, 1934.

6. Vincze St. Über den Minimalkreisring einer Eiline // Acta Sci. Math. (Szeged). 1947. V. 11. № 3. P. 133-138.

7. Vincze I. Über Kreisringe, die eine Eiline einschlissen // Studia Sci. Math. Hungar. 1974. V. 9. № $1 / 2$. P. $155-159$.

8. Kritikos N. Über konvexe Flachen und einschlissende Kugeln // Math. Ann. 1927. V. 96. P. 583-586.

9. Barany I. On the minimal ring containing the boundary of convex body // Acta Sci. Math. (Szeged). 1988. V. 52. №1/2. P. 93-100.

10. Zucco A. Minimal shell of a typical convex body // Proc. Amer. Math. Soc. 1990. V. 109. № 3. P. 797-802.

11. Иоффе А. Д., Тихомиров В. М. Теория экстремальных задач. М.: Наука, 1974.

12. Демьянов В.Ф., Васильев Л. В. Недифференцируемая оптимизация. М.: Наука, 1981.

13. Рокафеллар Р. Выпуклый анализ. М.: Мир, 1973.

14. Лейхтвейс K. Вьпуклые множества. М.: Наука, 1985.

15. Половинкин Е. С. Сильно выпуклый анализ // Матем. сб. 1996. Т. 187. № 2. С. 102-130.

16. Пиеничный Б. Н. Необходимые условия экстремума. М.: Наука, 1982.

17. Кларк Ф. Оптимизация и негладкий анализ. М.: Наука, 1988.

18. Дудов С.И. Субдифференцируемость и супердифференцируемость функции расстояния // Матем. заметки. 1997. Т. 61. № 4. С. 530-542.

19. Дудов С. И. Дифференцируемость по направлениям функции расстояния // Матем. сб. 1995. Т. 186. № 3. C. 29-52.

Саратовский государственный университет им. Н.Г. Черньшевского;

Саратовский социально-экономический государственный университет

E-mail: DudovSI@info.sgu.ru, Zlato@fort.ssea.runnet.ru
Поступила в редакцию 26.07.1999 